\title{
PRODUCCIÓN Y DESCOMPOSICIÓN DE HOJARASCA EN DIFERENTES CONDICIONES SUCESIONALES DEL BOSQUe DE PINO-ENCINO EN ChIAPAS, MÉXICO
}

\author{
Ana Guadalupe Rocha-Loredo y Neptalí Ramírez-Marcial ${ }^{1}$ \\ Departamento de Ecología y Sistemática Terrestres. Conservación de la Biodiversidad, \\ El Colegio de la Frontera Sur, Carretera Panamericana y Periférico Sur s/n, Colonia Ma. Auxiliadora, C.P. 29290, \\ San Cristóbal de Las Casas, Chiapas. \\ 1'Autor para la correspondencia. Correo-e: nramirezm@ecosur.mx
}

\begin{abstract}
Resumen: Se cuantificó la producción de hojarasca en comunidades sucesionales del boque de pino-encino en dos localidades de Chiapas y se evaluó simultáneamente la descomposición de hojarasca de siete especies (Alnus acuminata subsp. arguta, Chiranthodendron pentadactylon, Clethra suaveolens, Liquidambar styraciflua, Quercus crassifolia, Quercus sapotifolia y Pinus oocar$p a)$. Después de un año, la máxima acumulación de hojarasca se registró en el bosque de pino-encino $\left(7.59 \pm 0.29 \mathrm{Mg} \cdot h \mathrm{a}^{-1} \cdot \mathrm{año} \mathrm{O}^{-1}\right)$, seguido por el bosque de encino $\left(6.58 \pm 0.27 \mathrm{Mg} \cdot \mathrm{ha}^{-1} \cdot \mathrm{anno}^{-1}\right)$, el bosque de pino-encino-Liquidámbar $\left(5.33 \pm 0.18 \mathrm{Mg} \cdot \mathrm{ha}^{-1} \cdot \mathrm{año}^{-1}\right)$, el bosque de pino $\left(5.18 \pm 0.19 \mathrm{Mg} \cdot \mathrm{ha}^{-1} \cdot \mathrm{año}^{-1}\right)$ y el menor aporte anual de hojarasca se registró en las parcelas bajo restauración forestal

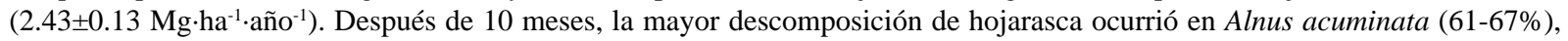
independientemente de la condición forestal; en el resto de las especies la descomposición varió de 34-52\% y no se detectaron diferencias entre las condiciones del bosque.
\end{abstract}

Palabras clave: Árboles nativos, bosque de Pinus-Quercus-Liquidambar, Chiranthodendron pentadactylon, lagunas de Montebello, materia orgánica, restauración forestal, trampas de hojarasca.

\begin{abstract}
Litter production was evaluated in successional pine-oak forests in two localities in Chiapas; simultaneously we assessed the leaf-litter decomposition of seven tree species (Alnus acuminata subsp. arguta, Chiranthodendron pentadactylon, Clethra suaveolens, Liquidambar styraciflua, Quercus crassifolia, Quercus sapotifolia and Pinus oocarpa). After 1-year, the highest litter production was recorded in pine-oak-forests $\left(7.59 \pm 0.29 \mathrm{Mg} \cdot \mathrm{ha}^{-1} \cdot \mathrm{year}^{-1}\right)$, followed by the oak forests $\left(6.58 \pm 0.27 \mathrm{Mg} \cdot \mathrm{ha}^{-1} \cdot \mathrm{year} \mathrm{r}^{-1}\right)$, pineoak-Liquidambar forests $\left(5.33 \pm 0.18 \mathrm{Mg} \cdot \mathrm{ha}^{-1} \cdot\right.$ year $\left.^{-1}\right)$, pine forest $\left(5.18 \pm 0.19 \mathrm{Mg} \cdot \mathrm{ha}^{-1} \cdot \mathrm{year}^{-1}\right)$, and the lowest annual litter production occurred in the plots under forest restoration $\left(2.43 \pm 0.13 \mathrm{Mg} \cdot \mathrm{ha}^{-1} \cdot \mathrm{year}^{-1}\right)$. After 10-mo, the highest rate of leaf-litter decomposition occurred in Alnus acuminata (61-67\%) regardless of the forest conditions and in the rest of the species, litter decomposition ranged from $34-52 \%$, but not significant differences between forest conditions were observed.

Key words: Chiranthodendron pentadactylon, forest restoration, litterbags, Montebello National Park, native trees, organic matter content, Pinus-Quercus-Liquidambar forest.
\end{abstract}

L a producción y descomposición de hojarasca son procesos en los que la materia orgánica se deposita y reduce en sus elementos constituyentes. Ambos procesos regulan la cantidad y contenido bioquímico de la materia orgánica producida en un ecosistema (Aber y Melillo, 1991) y son responsables de la formación de sustancias húmicas que contribuyen a la calidad/fertilidad del suelo (Berg y Mc-
Claugherty, 2008). La descomposición de la hojarasca está controlada por tres factores principales: el clima, la calidad de la hojarasca y la abundancia de organismos degradadores de la misma (Lavelle et al., 1993; Coûteaux et al., 1995; Aerts, 1997). En sentido muy amplio, la descomposición incluye mecanismos físicos, químicos y biológicos que transforman la materia orgánica dentro de formas cada vez 
más estables. En el primer caso, puede darse la fractura o fragmentación de estructuras grandes en pequeñas a través del viento, animales u otras estructuras, mediante la lixiviación de compuestos solubles en agua o transportados en ella; entre las transformaciones químicas se reconoce a la oxidación y a la condensación, en tanto que en los mecanismos biológicos se incluye a la ingestión y digestión, junto con actividad enzimática extracelular (Aber y Melillo, 1991; Berg y McClaugherty, 2008). El conocimiento de ambos procesos constituye un paso necesario para el manejo de sistemas productivos (Wedderburn y Carter, 1999; Sangha et al., 2006), tanto como para la restauración de paisajes forestales (Borders et al., 2006).

Aunque existe información de la producción y descomposición de hojarasca para varios ecosistemas forestales tropicales (por ej., Babbar y Ewel, 1989; Sundarapandian y Swamy, 1999; Epstein et al., 2002; Lawrence, 2005) la información para los bosques tropicales de montaña es relativamente menor. En México se han documentado tasas de descomposición de especies arbóreas (por ej., Bracho y Puig, 1987; Álvarez-Sánchez y Becerra, 1996; WilliamsLinera y Tolome, 1996; Del Valle-Arango, 2003), pero estudios que evalúen simultáneamente estas variables en diferentes comunidades forestales o a lo largo de gradientes sucesionales son mucho más escasos (Negrete-Yankelevich, 2004). A pesar del reconocimiento de los bosques tropicales de montaña como megadiversos, la distribución reducida y cada vez más fragmentada sigue siendo una de las mayores amenazas para su mantenimiento y conservación (Golicher et al., 2008). En los fragmentos forestales remanentes se ha observado una disminución en el número de individuos adultos reproductivos de varias de las especies arbóreas autóctonas (Ramírez-Marcial et al., 2001, 2005). Las especies del interior en los bosques de encino y de pino-encino han sido las más severamente afectadas con la extinción local (Camacho-Cruz et al., 2000; Ramírez-Marcial et al., 2006). Muchas de las especies de éstos bosques se consideran sucesionalmente intermedias o tardías, por lo que requieren de la preexistencia de una cubierta arbórea para asegurar un buen establecimiento (Ramírez-Marcial et al., 2006). Una de estas condiciones previas para el establecimiento de plántulas es la "calidad" del hábitat como nicho de regeneración, en el cual la cantidad y tipo de hojarasca puede afectar las probabilidades de colonización de una especie.

En el presente trabajo se evaluó la acumulación de hojarasca comparativamente en cinco condiciones sucesionales de los bosques de pino-encino en Los Altos de Chiapas. Simultáneamente se cuantificó la tasa de descomposición de la hojarasca de siete especies de árboles nativos con el objetivo de conocer si hay diferencias entre ellas y si la respuesta varía entre las condiciones del bosque incluidas en el estudio.

\section{Materiales y métodos}

El presente trabajo se realizó en dos localidades: Merced Bazom, municipio de Huistán y el Parque Nacional Lagunas de Montebello, municipio de La Trinitaria, ubicadas respectivamente en el centro y límite oriental de la región de la Altiplanicie Central de Chiapas. Las localidades difieren en características físicas y estructurales (Cuadro 1), pero ambas comparten una larga historia de uso del suelo que ha dado como resultado un gradiente sucesional del bosque de pino-encino, que incluye desde etapas maduras, representadas por el bosque de encino y pino-encino, los bosques secundarios de pino-encino-Liquidámbar y los bosques más perturbados como el bosque de pino y áreas de vegetación secundaria arbustiva, resultante del patrón continuo de

Cuadro1. Descripción de las principales características físicas y estructurales de las comunidades forestales consideradas en el estudio.

\begin{tabular}{|c|c|c|c|c|c|}
\hline Localidad & Tipo de Vegetación & $\begin{array}{l}\text { Altitud } \\
\text { (msnm) }\end{array}$ & $\begin{array}{c}\text { Área basal } \\
\text { dominantes } \\
\left(\mathrm{m}^{2} \cdot \mathrm{ha}^{-1}\right)\end{array}$ & Especies & $\begin{array}{l}\text { Tipo de } \\
\text { suelos }\end{array}$ \\
\hline \multirow[t]{3}{*}{ Merced Bazom } & Bosque de encino (BE) & 2460 & $54 \pm 6.2^{\mathrm{a}}$ & $\begin{array}{l}\text { Quercus crassifolia, } \\
\text { Q. rugosa, Q. laurina }\end{array}$ & $\begin{array}{l}\text { Luvisoles y } \\
\text { Rendzinas }\end{array}$ \\
\hline & Bosque de pino-encino (BPE) & 2500 & $60 \pm 4.6^{\mathrm{a}}$ & $\begin{array}{l}\text { Quercus crassifolia, } \\
\text { Pinus pseudostrobus }\end{array}$ & Luvisol \\
\hline & Bosque de pino (BP) & 2480 & $39 \pm 5.9^{a}$ & Pinus pseudostrobus & Rendzina \\
\hline \multirow[t]{2}{*}{$\begin{array}{l}\text { Parque Nacional } \\
\text { Lagunas de } \\
\text { Montebello }\end{array}$} & $\begin{array}{c}\text { Bosque de } \\
\text { pino-encino-liquidámbar } \\
\text { (BPEL) }\end{array}$ & 1600 & $37 \pm 4.4^{b}$ & $\begin{array}{c}\text { Pinus oocarpa, } \\
\text { Quercus sapotifolia }\end{array}$ & $\begin{array}{l}\text { Rendzinas y } \\
\text { Vertisoles }\end{array}$ \\
\hline & $\begin{array}{c}\text { Parcelas en } \\
\text { restauración (PR) }\end{array}$ & 1560 & $11 \pm 4.7^{\mathrm{b}}$ & $\begin{array}{l}\text { Pinus oocarpa, } \\
\text { Clethra suaveolens }\end{array}$ & Rendzinas \\
\hline
\end{tabular}

a González-Espinosa et al. 1991, 2006; ' Rodríguez-Sánchez 2006. 
perturbación antropogénica (Galindo-Jaimes et al., 2002; González-Espinosa et al., 1991, 2006).

En la localidad de Merced Bazom se delimitaron seis parcelas de 0.25 ha $(50 \times 50 \mathrm{~m})$, considerando dos parcelas para cada una de las siguientes comunidades forestales: (1) bosque de encino (BE), (2) bosque de pino-encino (BPE) y (3) bosque de pino (BP). En la localidad de Montebello, se seleccionaron otras dos comunidades forestales: (4) bosque de pino-encino-liquidámbar (BPEL) y (5) parcelas bajo restauración (PR) que corresponden a un ensayo de enriquecimiento de bosques secundarios con 16 especies arbóreas nativas, establecido durante el verano de 2003 (Ortíz-Aguilar, 2006). Al igual que en Merced Bazom, se establecieron dos parcelas de 0.25 ha en cada una de las dos comunidades forestales. Para cuantificar la acumulación de hojarasca se colocaron 20 trampas captadoras dentro de cada una de las 10 parcelas. Las trampas consistieron de armazones metálicos con forma cúbica $(90 \times 30 \times 30 \mathrm{~cm})$ y forrados en todos los lados excepto en la cara superior (área de $90 \times 30 \mathrm{~cm}$ ) con tela de propileno de $2 \mathrm{~mm}^{2}$ de luz. Las trampas se colocaron horizontalmente y elevadas a $30 \mathrm{~cm}$ de la superficie del suelo. Las trampas fueron distribuidas sistemáticamente a cada $10 \mathrm{~m}$ entre sí dentro de cada parcela (200 trampas en total para las 10 parcelas). La hojarasca se recolectó mensualmente a lo largo de un año (marzo de 2005-febrero de 2006). De cada trampa se obtuvo el peso fresco y el peso seco después de poner las muestras durante 48 horas a $70^{\circ}$ $\mathrm{C}$ en un horno de convección. Para calcular la cantidad de hojarasca por unidad de área, el peso de hojarasca de cada trampa se dividió por el área de la trampa $\left(0.27 \mathrm{~m}^{2}\right)$, se multiplicó por el número de trampas (20) por parcela y se extrapoló a una hectárea, expresando los valores en megagramos de hojarasca por hectárea por mes $\left(\mathrm{Mg} \cdot \mathrm{ha}^{-1} \cdot \mathrm{mes}^{-1}\right)$. Para distinguir la aportación de distintos componentes de la biomasa acumulada en la hojarasca, se tomó al azar el contenido de cinco trampas por cada parcela y se separó en cinco categorías: hojas, material leñoso (ramas y corteza), flores, frutos y no identificable (partes muy pequeñas que no fue posible separar o identificar su origen dentro de las categorías anteriores).

Para evaluar la tasa de descomposición de la hojarasca de algunas especies seleccionadas, se utilizó el método de "bolsas de descomposición" (litterbag method; Bärlocher, 2005). Se usaron bolsas de polipropileno negro de $17 \times 25 \mathrm{~cm}$ con apertura de $2 \mathrm{~mm}^{2}$ conteniendo una cantidad constante de 10 g de hojarasca en peso seco. Debido a que la composición de las especies dominantes es distinta entre las localidades, se decidió utilizar especies diferentes en cada localidad. En Merced Bazom se colocaron 20 bolsas por parcela dentro de los tres tipos de bosque (BE, BPE y BP) con $10 \mathrm{~g}$ de hojarasca de Alnus acuminata Kunth. subsp. arguta (Schltdl.) Furlow, Chiranthodendron pentadactylon Larreat., Quercus crassifolia Humb. \& Bonpl. y Pinus oocarpa Schiede ex Schltdl. En Montebello, las especies utilizadas fueron Cle- thra suaveolens Turcz., Liquidambar styraciflua L., Quercus sapotifolia Liebm. y Pinus oocarpa, con 20 bolsas por cada especie por parcela en el BPEL y PR. Un total de 800 bolsas fueron empleadas para todo el diseño experimental. Cada bolsa se colocó superficialmente muy cerca de cada vértice de cada trampa de hojarasca. Posteriormente, se retiraron al azar cinco bolsas de cada especie por parcela en diferentes momentos $(60,120,210$ y 300 días); las bolsas se secaron durante 48 horas a $70^{\circ} \mathrm{C}$ en un horno de convección para obtener el peso seco residual de cada muestra. Con este valor se obtuvo el porcentaje de pérdida de la biomasa inicial equivalente a la descomposición. Previo a la colocación de las bolsas de descomposición y al final del experimento, se analizó para cada especie la concentración de Carbono y Nitrógeno por combustión de la muestra mediante un Autoanalizador LECO CHN-1000 (Anderson e Ingram, 1993).

Para analizar la variación en la acumulación de hojarasca (peso seco) se aplicó un análisis de varianza bifactorial (Quinn y Keough, 2002), en donde la condición del bosque y el mes de evaluación fueron los dos factores fijos. Cuando se detectaron diferencias significativas $(p<0.05)$ entre los factores principales se aplicaron pruebas de comparación de medias con el método de Bonferroni. Utilizamos el modelo de Olson (1963) para obtener el coeficiente de descomposición de la hojarasca $(k)$, el cual estima la tasa de pérdida de biomasa sobre una base anual. Este modelo asume una función exponencial negativa del tipo $x_{t} / x_{0}=\mathrm{e}^{-\mathrm{kt}}$, donde $x_{0}=$ la cantidad original de la muestra $(10 \mathrm{~g})$ y $x_{t}$ es la cantidad de muestra remanente en el tiempo $t$ (en años). Calculamos el valor de $k$ a los 60, 120, 210 y 300 días, pero para el análisis utilizamos el promedio de las cuatro estimaciones por especie dentro de cada tipo de vegetación. De esta manera, se utilizaron los datos de prácticamente todas las bolsas de descomposición por localidad ( $\mathrm{N}=470$ en Merced Bazom y $\mathrm{N}=318$ en Montebello; 12 muestras extraviadas) y así removimos el efecto del tiempo de cosecha de cada conjunto de 5 trampas (Cleveland et al., 2006). Las diferencias entre los coeficientes de descomposición de hojarasca $(k)$ fueron entonces analizadas separadamente por localidad mediante un análisis de varianza bifactorial, donde el tipo de bosque y la especie fueron los factores principales. Cuando se detectaron diferencias significativas se utilizaron pruebas de comparación de medias con el método de Bonferroni. Todos los análisis se realizaron con el programa $\mathrm{R}$, versión 2.6.2 (R Development Core Team, 2008).

\section{Resultados}

Producción de hojarasca. A lo largo del año de evaluación, la producción de hojarasca varió significativamente entre los tipos de bosque $\left(F_{4}, 2120=113.24, p<0.001\right)$, el mes de evaluación $\left(F_{11}, 2120=32.89, p<0.001\right)$ y la interacción entre ambos factores principales $\left(F_{44}, 2120=9.31, p<0.001\right.$; figura 1). La mayor acumulación anual de hojarasca se registró en el 


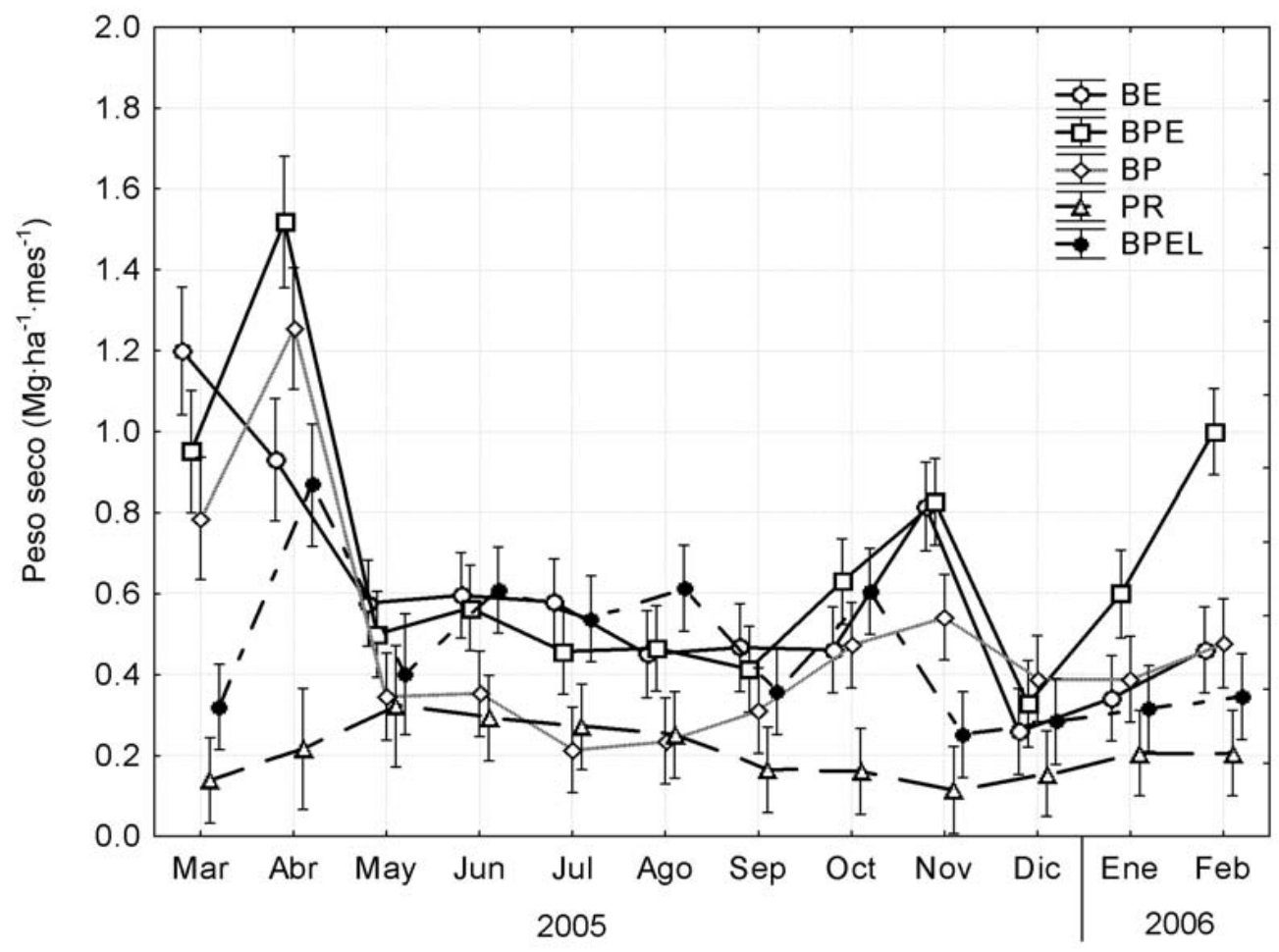

Figura 1. Acumulación mensual de hojarasca (promedio \pm e.e., $\mathrm{Mg} \cdot \mathrm{ha}^{-1} \cdot \mathrm{mes}^{-1}$ ) durante marzo de 2005 -febrero de 2006 en cinco comunidades forestales en Chiapas: $\mathrm{BE}=$ bosque de encino, $\mathrm{BP}=$ bosque de pino, $\mathrm{BPE}=$ bosque de pino-encino, $\mathrm{BPEL}=$ bosque de pino-encinoliquidámbar y $\mathrm{PR}=$ parcelas bajo restauración forestal.

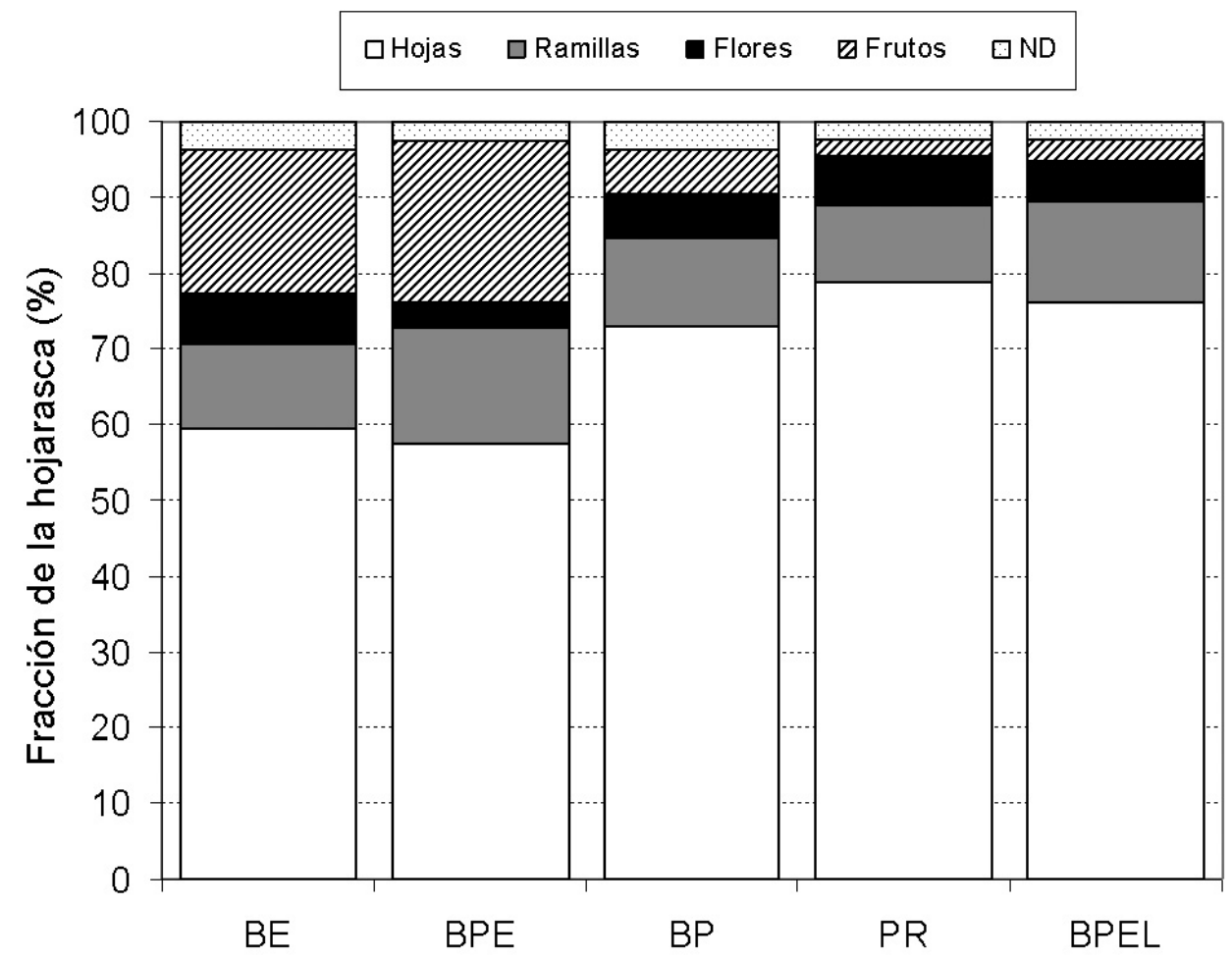

Figura 2. Representación porcentual de los diferentes componentes de la hojarasca registrados en las cinco comunidades forestales en Chiapas: $\mathrm{BE}=$ bosque de encino, $\mathrm{BP}=$ bosque de pino, $\mathrm{BPE}=$ bosque de pino-encino, $\mathrm{BPEL}=$ bosque de pino-encino-liquidámbar y $\mathrm{PR}=$ parcelas bajo restauración forestal. ND = material no determinado. 
a) Merced Bazom
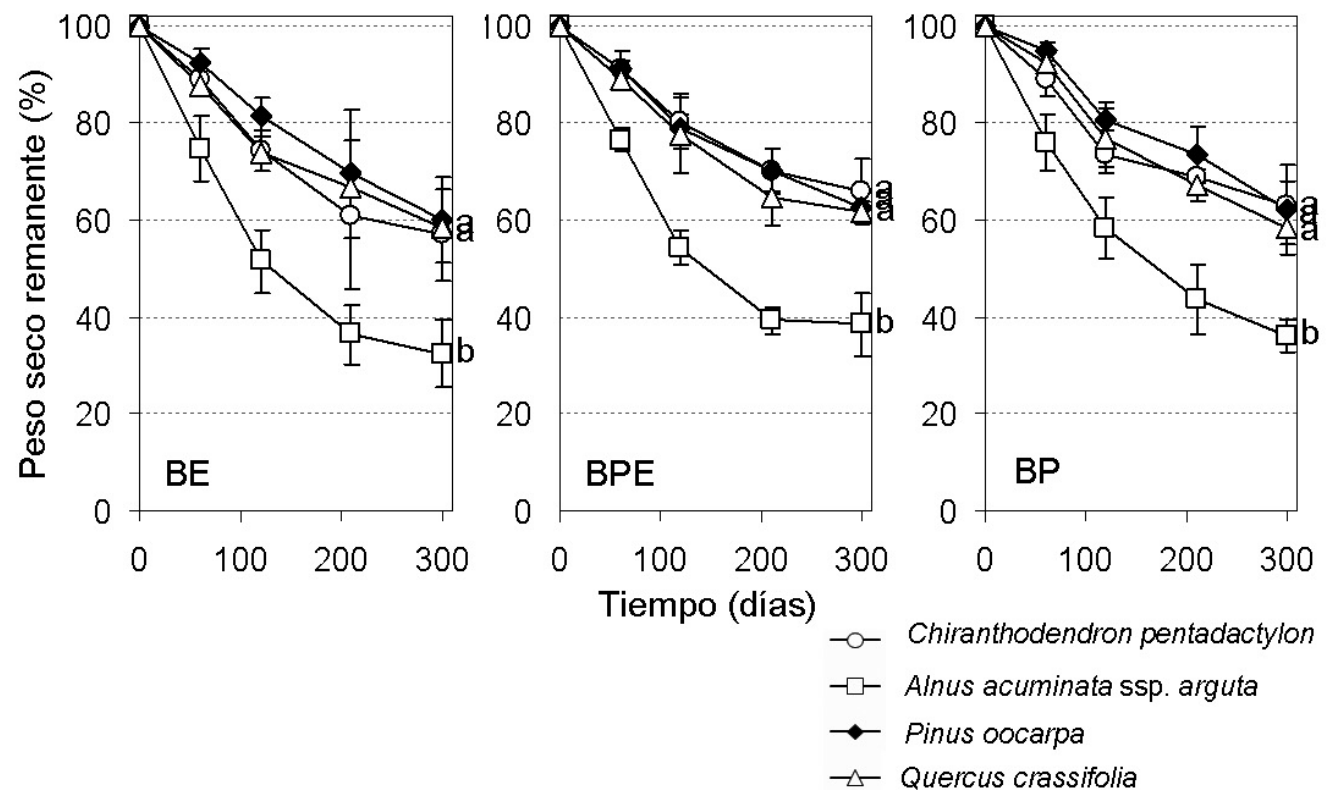

b) Lagunas de Montebello

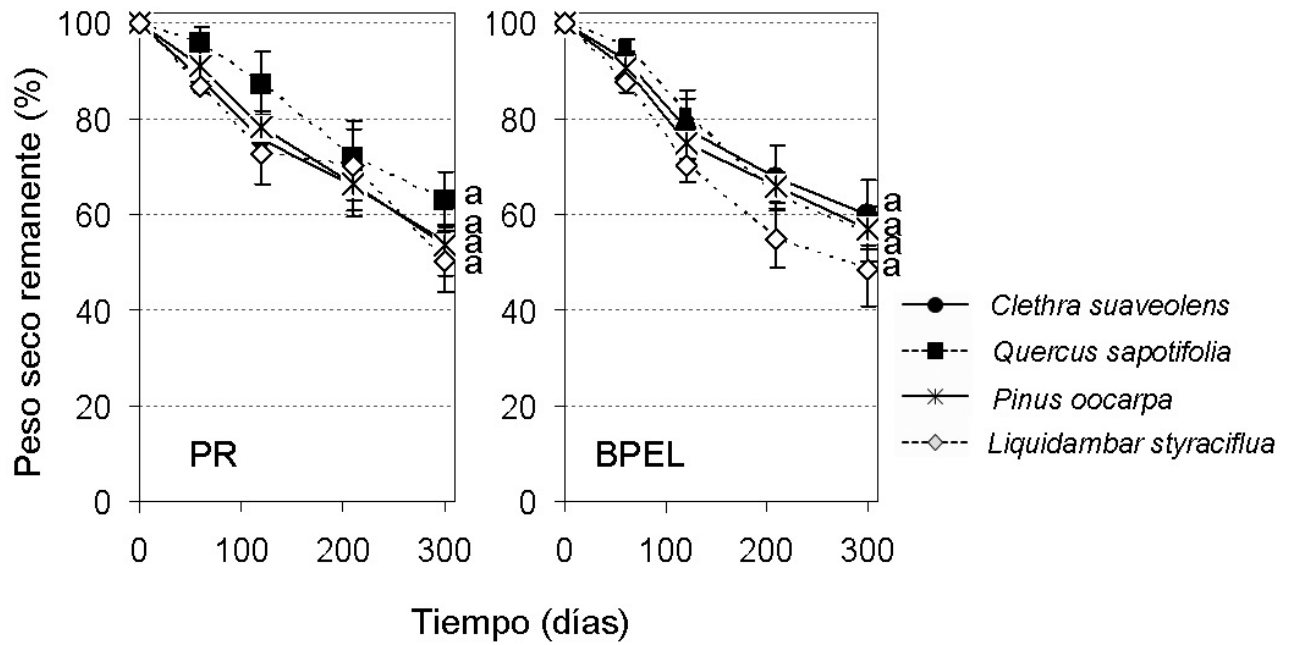

Figura 3. Porcentaje de peso seco remanente (promedio \pm e.e.) en bolsas de descomposición de hojarasca para diferentes especies dispuestas en distintas condiciones forestales en dos localidades de Chiapas, (a) Merced Bazom: BE $=$ bosque de encino, BPE $=$ bosque de pino-encino, BP = bosque de pino y (b) Parque Nacional Lagunas de Montebello: BPEL= bosque de pino-encino-liquidámbar y PR = parcelas bajo restauración forestal. Las letras diferentes al final de cada curva denotan diferencias significativas de las especies dentro de cada condición de bosque después del ANOVA $(p<0.001)$.

BPE $\left(7.59 \pm 0.29 \mathrm{Mg} \cdot \mathrm{ha}^{-1} \cdot \mathrm{año}^{-1}\right)$, seguida del BE $(6.58 \pm 0.27$

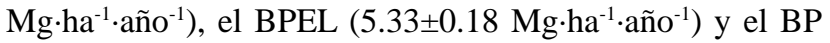
$\left(5.18 \pm 0.19 \mathrm{Mg} \cdot \mathrm{ha}^{-1} \cdot \mathrm{año}^{-1}\right)$. La cantidad de hojarasca registrada en las parcelas bajo restauración forestal (PR) fue significativamente inferior $\left(2.43 \pm 0.13 \mathrm{Mg} \cdot \mathrm{ha}^{-1} \cdot \mathrm{año}^{-1}\right)$ al resto de las otras condiciones forestales. Sobre la base temporal, se observó un pico de máxima caída de hojarasca durante el inicio del periodo de muestreo (marzo-abril de 2005) en la mayoría de los bosques analizados; sin embargo, hubo otro pulso de alta acumulación de hojarasca entre octubre y noviembre del mismo año atribuible al paso del huracán Stan. La menor cantidad y variación en la acumulación mensual de hojarasca se registró en las parcelas bajo restauración (0.12-0.34 $\mathrm{Mg} \cdot \mathrm{ha}^{-1} \cdot \mathrm{mes}^{-1}$; figura 1).

En general, la fracción principal de la hojarasca lo constituyó las hojas (72-90\%), seguido de ramas y corteza (5$12 \%)$, frutos $(0.75-12 \%)$, flores $(2-3 \%)$ y material no identificado (1-2\%; figura 2). Esta variación en la distribución de 
los componentes de la hojarasca resultó significativamente diferente entre los tipos de vegetación $\left(F_{4}, 1971=230.26\right.$; $p<0.001)$ así como entre la interacción con el tiempo de evaluación $\left(F_{40}, 1971=3.14 ; p<0.001\right)$. Por lo general, la mayor presencia de estructuras reproductivas en el BE y BPE se correspondió con el estado fenológico de las especies dominantes, donde las flores o restos de ellas fueron más frecuentes en abril y los frutos en noviembre (datos no presentados).

Descomposición de hojarasca. La descomposición de hojarasca por localidad mostró algunas diferencias entre las especies dentro de las condiciones de bosque (figura 3). En Merced Bazom, el porcentaje de peso remanente varió significativamente entre las especies $\left(F_{3}, 942=21.96 ; p<0.001\right)$, pero no entre los tipos de bosque $\left(F_{2}, 942=0.81 ; p>0.05\right)$. En cambio, en Montebello, no se detectaron diferencias en- tre los factores analizados $\left(F_{1}, 626=0.629 ; p>0.05\right.$ para la condición de bosque y $F_{3}, 626=1.99 ; p>0.05$ para la especie). En cambio, cuando se realizó el análisis de la variación en los coeficientes de descomposición $(k)$ se encontraron diferencias significativas entre las especies de Merced Bazom $\left(F_{3}, 458=136.71 ; p<0.001\right)$ y entre los tipos de bosque $\left(F_{2}, 458=7.18 ; p=0.001\right)$ y la interacción de ambos factores $\left(F_{6}, 458=2.43 ; p=0.025\right)$.

Por otro lado, en la localidad de Montebello, únicamente se detectaron diferencias significativas entre las especies $\left(F_{3}\right.$, $307=19.79 ; p<0.001)$ y en la interacción entre la condición de bosque y las especies $\left(F_{3}, 307=4.61 ; p=0.004\right)$, pero no entre los dos tipos de bosque $\left(F_{1}, 307=0.073 ; p=0.78\right)$. Notablemente, Alnus acuminata subsp. arguta tuvo el valor más alto de la constante de descomposición en los tres tipos de bosque evaluados en Merced Bazom (BE: $k=1.74 \pm$ 0.114; BPE: $k=1.44 \pm 0.082$; BP: $k=1.40 \pm 0.087$; figura

Cuadro 2. Concentración de Carbono, Nitrógeno y relación $\mathrm{C} / \mathrm{N}$ (promedio \pm e.e) al inicio y después de 10 meses presente en la hojarasca de siete especies de árboles evaluados en: $\mathrm{BE}=$ bosque de encino, $\mathrm{BP}=$ bosque de pino, $\mathrm{BPE}=$ bosque de pino-encino, $\mathrm{BPEL}=$ bosque de pinoencino-liquidámbar y PR = parcelas bajo restauración forestal. Para cada especie, letras diferentes (en superíndice) a la derecha de cada columna representa diferencias significativas $(p<0.05)$ posterior al ANOVA para los valores iniciales y finales entre las condiciones forestales.

\begin{tabular}{|c|c|c|c|c|}
\hline $\begin{array}{l}\text { Localidad } \\
\text { Especie }\end{array}$ & Condición & Carbono (\%) & Nitrógeno (\%) & Relación C/N \\
\hline $\begin{array}{l}\text { Merced Bazom } \\
\text { A. acuminata subsp. arguta }\end{array}$ & $\begin{array}{r}\text { Inicial } \\
\text { BE } \\
\text { BP } \\
\text { BPE }\end{array}$ & $\begin{array}{l}52.4 \pm 0.6^{\mathrm{a}} \\
51.4 \pm 2.1^{\mathrm{a}} \\
49.6 \pm 0.2^{\mathrm{a}} \\
52.1 \pm 0.7^{\mathrm{a}}\end{array}$ & $\begin{array}{l}1.6 \pm 0.02^{\mathrm{a}} \\
1.3 \pm 0.02^{\mathrm{b}} \\
1.8 \pm 0.09^{\mathrm{a}} \\
1.4 \pm 0.04^{\mathrm{b}}\end{array}$ & $\begin{array}{l}31.5 \pm 0.6^{\mathrm{b}} \\
38.5 \pm 0.8^{\mathrm{a}} \\
27.4 \pm 1.3^{\mathrm{c}} \\
36.3 \pm 0.6^{\mathrm{a}}\end{array}$ \\
\hline C. pentadactylon & $\begin{array}{r}\text { Inicial } \\
\text { BE } \\
\text { BPE } \\
\text { BP }\end{array}$ & $\begin{array}{r}50.0 \pm 0.15^{\mathrm{a}} \\
47.7 \pm 1.55^{\mathrm{a}} \\
44.5 \pm 1.7^{\mathrm{a}} \\
50.7 \pm 1.3^{\mathrm{a}}\end{array}$ & $\begin{array}{l}1.0 \pm 0.05^{\mathrm{a}} \\
1.8 \pm 0.57^{\mathrm{a}} \\
1.2 \pm 0.01^{\mathrm{a}} \\
1.4 \pm 0.25^{\mathrm{a}}\end{array}$ & $\begin{array}{l}47.8 \pm 2.6^{\mathrm{a}} \\
29.7 \pm 8.7^{\mathrm{a}} \\
37.4 \pm 1.1^{\mathrm{a}} \\
37.3 \pm 7.3^{\mathrm{a}}\end{array}$ \\
\hline Q. crassifolia & $\begin{array}{r}\text { Inicial } \\
\text { BE } \\
\text { BPE } \\
\text { BP }\end{array}$ & $\begin{array}{l}50.9 \pm 0.39^{\mathrm{a}} \\
52.7 \pm 0.05^{\mathrm{b}} \\
53.4 \pm 0.04^{\mathrm{b}} \\
53.1 \pm 0.02^{\mathrm{b}}\end{array}$ & $\begin{array}{c}1.6 \pm 0.07^{\mathrm{b}} \\
2.5 \pm 0.03^{\mathrm{a}} \\
1.9 \pm 0.42^{\mathrm{ab}} \\
1.6 \pm 0.03^{\mathrm{ab}}\end{array}$ & $\begin{array}{l}32.5 \pm 1.5^{\mathrm{a}} \\
20.9 \pm 0.3^{\mathrm{a}} \\
28.2 \pm 5.8^{\mathrm{a}} \\
32.8 \pm 0.5^{\mathrm{a}}\end{array}$ \\
\hline P. oocarpa & $\begin{array}{r}\text { Inicial } \\
\text { BE } \\
\text { BPE } \\
\text { BP }\end{array}$ & $\begin{array}{r}52.5 \pm 0.10^{\mathrm{a}} \\
49.1 \pm 2.15^{\mathrm{a}} \\
51.1 \pm 2.8^{\mathrm{a}} \\
51.0 \pm 0.70^{\mathrm{a}}\end{array}$ & $\begin{array}{l}1.2 \pm 0.15^{\mathrm{a}} \\
1.4 \pm 0.05^{\mathrm{a}} \\
2.1 \pm 0.66^{\mathrm{a}} \\
2.5 \pm 0.08^{\mathrm{a}}\end{array}$ & $\begin{array}{c}43.1 \pm 5.1^{\mathrm{a}} \\
35.5 \pm 0.2^{\mathrm{b}} \\
26.6 \pm 7.1^{\mathrm{bc}} \\
20.0 \pm 0.3^{\mathrm{c}}\end{array}$ \\
\hline $\begin{array}{l}\text { Lagunas Montebello } \\
\text { C. suaveolens }\end{array}$ & $\begin{array}{r}\text { Inicial } \\
\text { BPEL } \\
\text { PR }\end{array}$ & $\begin{array}{r}49.9 \pm 0.26^{\mathrm{a}} \\
53.2 \pm 1.5^{\mathrm{a}} \\
53.6 \pm 0.55^{\mathrm{a}}\end{array}$ & $\begin{array}{l}1.3 \pm 0.12^{\mathrm{a}} \\
1.9 \pm 0.48^{\mathrm{a}} \\
1.5 \pm 0.01^{\mathrm{a}}\end{array}$ & $\begin{array}{l}39.3 \pm 3.99^{\mathrm{a}} \\
29.1 \pm 7.88^{\mathrm{a}} \\
36.1 \pm 0.25^{\mathrm{a}}\end{array}$ \\
\hline L. styraciflua & $\begin{array}{r}\text { Inicial } \\
\text { BPEL } \\
\text { PR }\end{array}$ & $\begin{array}{l}48.6 \pm 0.31^{\mathrm{a}} \\
48.6 \pm 0.25^{\mathrm{a}} \\
47.1 \pm 0.85^{\mathrm{a}}\end{array}$ & $\begin{array}{r}1.6 \pm 0.04^{\mathrm{a}} \\
1.32 \pm 0.05^{\mathrm{b}} \\
1.20 \pm 0.02^{\mathrm{b}}\end{array}$ & $\begin{array}{l}29.3 \pm 0.5^{\mathrm{b}} \\
36.9 \pm 1.7^{\mathrm{a}} \\
39.4 \pm 1.5^{\mathrm{a}}\end{array}$ \\
\hline Q. sapotifolia & $\begin{array}{r}\text { Inicial } \\
\text { BPEL } \\
\text { PR }\end{array}$ & $\begin{array}{l}51.4 \pm 0.44^{\mathrm{a}} \\
52.1 \pm 0.55^{\mathrm{a}} \\
52.4 \pm 0.80^{\mathrm{a}}\end{array}$ & $\begin{array}{l}0.8 \pm 0.01^{\mathrm{b}} \\
2.4 \pm 0.03^{\mathrm{a}} \\
2.5 \pm 0.05^{\mathrm{a}}\end{array}$ & $\begin{array}{r}60.8 \pm 0.76^{a} \\
21.7 \pm 0.5^{b} \\
20.9 \pm 0.1^{b}\end{array}$ \\
\hline P. oocarpa & $\begin{array}{r}\text { Inicial } \\
\text { BPEL } \\
\text { PR }\end{array}$ & $\begin{array}{l}52.5 \pm 0.10^{\mathrm{a}} \\
52.0 \pm 0.00^{\mathrm{a}} \\
53.0 \pm 0.70^{\mathrm{a}}\end{array}$ & $\begin{array}{l}1.2 \pm 0.15^{\mathrm{a}} \\
1.5 \pm 0.02^{\mathrm{a}} \\
1.9 \pm 0.46^{\mathrm{a}}\end{array}$ & $\begin{array}{l}43.1 \pm 5.1^{\mathrm{a}} \\
34.1 \pm 0.5^{\mathrm{b}} \\
28.1 \pm 6.2^{\mathrm{c}}\end{array}$ \\
\hline
\end{tabular}




\section{a) Merced Bazom}

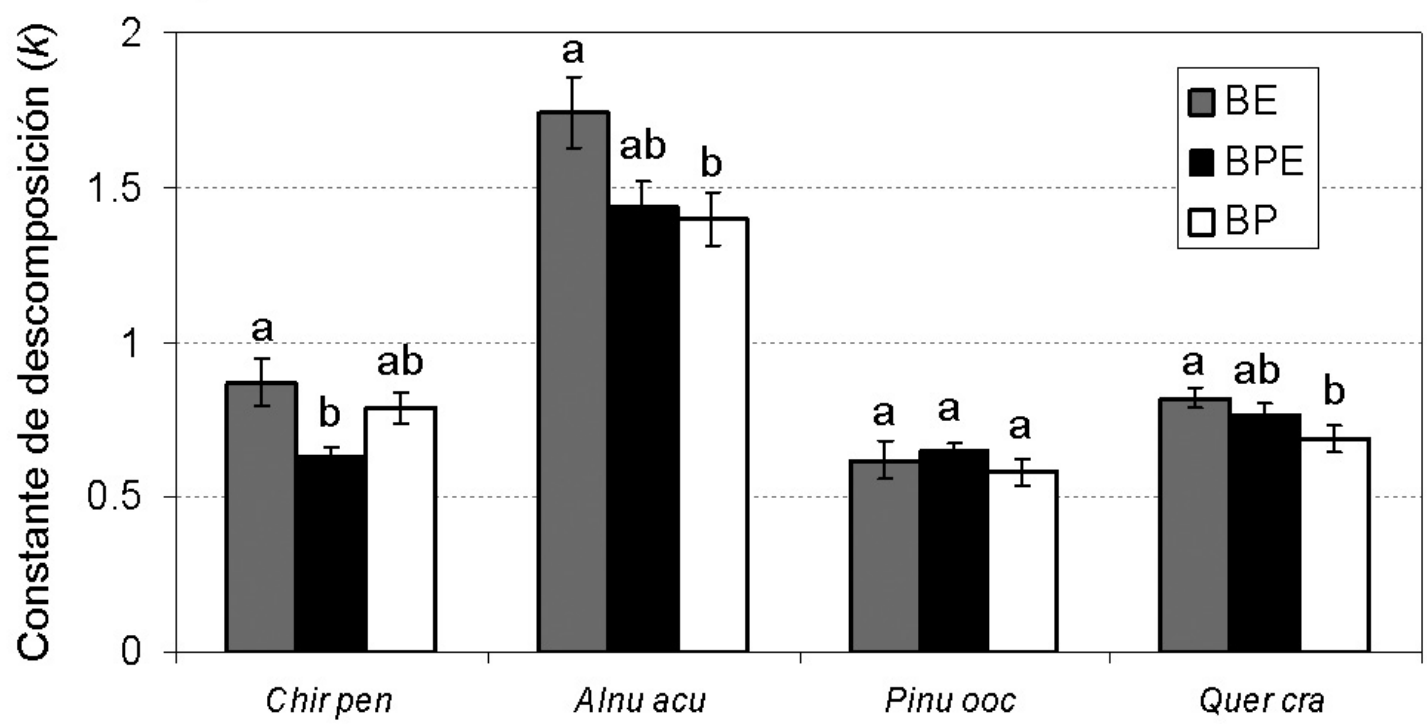

b) Lagunas de Montebello

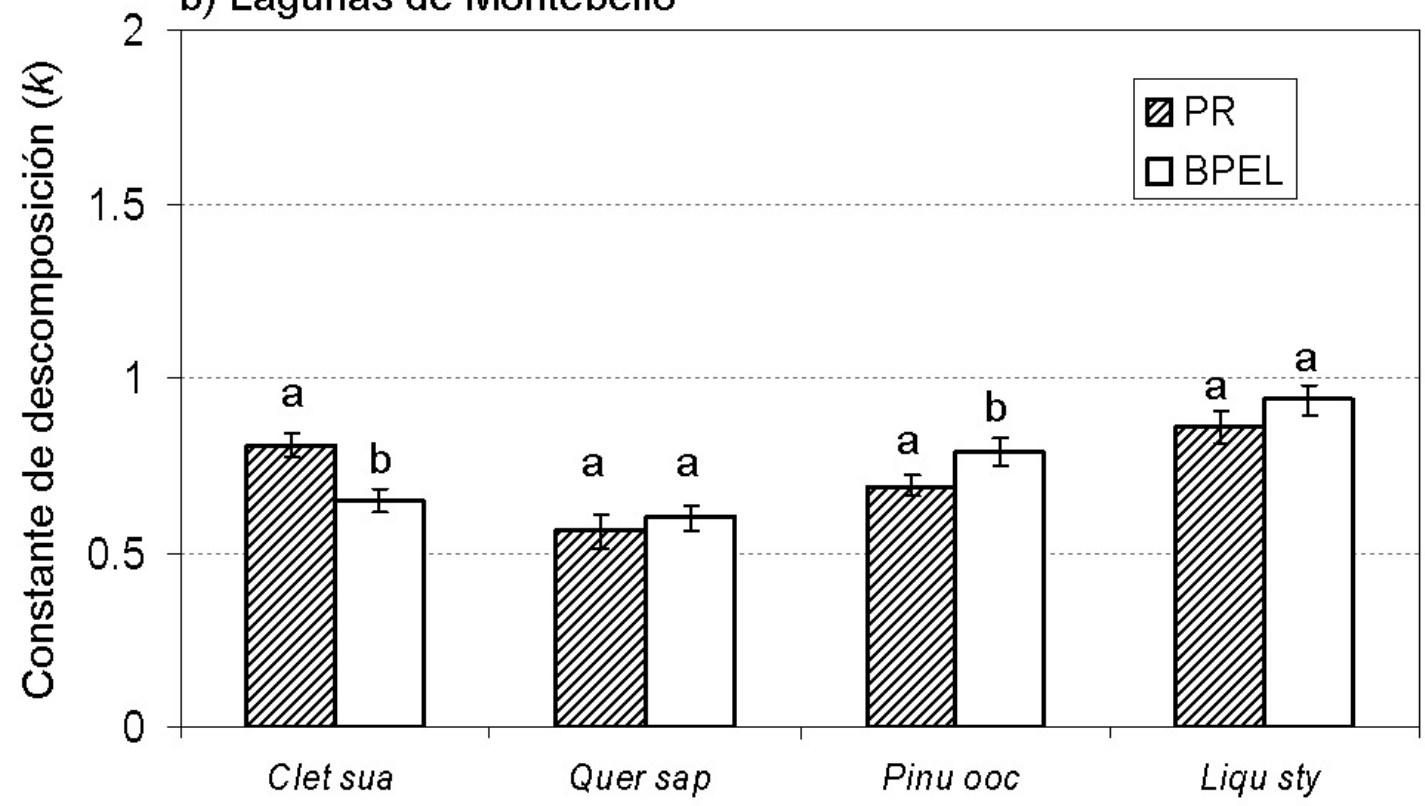

Figura 4. Coeficiente de la tasa de descomposición de la hojarasca ( $k$ ) para distintas especies en (a) bosque de encino (BE), bosque de pino-encino (BPE) y bosque de pino (BP) en Merced Bazom y (b) bosque de pino-encino-liquidámbar (BPEL) y en parcelas bajo restauración forestal (PR) en el Parque Nacional Lagunas de Montebello. Las especies utilizadas son: Alnu acu =Alnus acuminata subsp. arguta, Chir pen = Chiranthodendron pentadactylon, Pinu ooc $=$ Pinus oocarpa y Quer cra = Quercus crassifolia, Clet sua =Clethra suaveolens, Liqu sty = Liquidambar styraciflua, Pinu ooc = Pinus oocarpa y Quer sap = Quercus sapotifolia . Ver materiales y métodos para la explicación del cálculo de $k$. Para cada especie, las letras diferentes arriba de cada barra denotan diferencias significativas $(p<0.001)$ entre las condición de bosque comparados. 
4). En cambio, la velocidad de descomposición fue menos marcada entre las especies analizadas en Montebello, destacando Liquidambar styraciflua con el mayor valor de $k$ en ambas condiciones forestales (PR: $k=0.86 \pm 0.045$; BPEL: $k=0.94 \pm 0.043)$ y Quercus sapotifolia la de menor valor de $k$ (PR: $k=0.56 \pm 0.048$; BPEL: $k=0.60 \pm 0.036$; figura 4).

Concentración de Carbono, Nitrógeno y relación C/N en la hojarasca. La concentración inicial y final de $\mathrm{C}, \mathrm{N}$ y la relación de $\mathrm{C} / \mathrm{N}$ para todas las especies evaluadas se muestra en el Cuadro 2. El porcentaje inicial de $\mathrm{C}, \mathrm{N}$ y la relación entre $\mathrm{C} / \mathrm{N}$ varió entre las especies en cada localidad (50-52.5\% de C, $1-1.6 \%$ de $\mathrm{N}$ y 31.5-47.8\% de la relación $\mathrm{C} / \mathrm{N}$ en Merced Bazom; 48.6-52.5\% de C, 0-8-1.6\% de N y $29.3-60.8 \%$ de la relación C/N en Montebello, Cuadro 2). De esta manera, las concentraciones iniciales de dichos elementos resultaron significativamente diferentes entre las especies (Carbono: $F_{7}, 16=117.9, p<0.001$; Nitrógeno: $F_{7}$, $16=65.61, p<0.001$ y relación $\mathrm{C} / \mathrm{N}: F_{7}, 16=74.19, p<$ 0.001). Sin embargo, al final del experimento (10 meses), la concentración de estos elementos en Chiranthodendron pentadactylon no cambió entre las condiciones comparadas en Merced Bazom ni para Clethra suaveolens en Montebello (Cuadro 2). En cambio, el contenido de Nitrógeno y la relación $\mathrm{C} / \mathrm{N}$ mostraron diferencias significativas entre las condiciones del bosque para Alnus acuminata subsp. arguta, y Quercus sapotifolia (Cuadro 2).

\section{Discusión}

Los resultados de la captura de hojarasca indicaron una variación significativa en la cantidad de hojarasca entre las cinco condiciones forestales estudiadas. Esto puede explicarse por las diferencias en la estructura y composición florística a lo largo de la ruta sucesional. El incremento en la acumulación de hojarasca con la edad sucesional de un sitio se atribuye a la mayor producción de biomasa viva y al incremento en la caída de biomasa foliar (Berg y McClaugherty, 2008). Debido a que el bosque de pino-encino (BPE) y el bosque de encino (BE) se consideran sucesionalmente más desarrollados dentro de la región de estudio (González-Espinosa et al., 1991, 2006), no es extraño que hayan resultado ser las condiciones con los valores más altos de producción de hojarasca pues son las dos condiciones con la mayor área basal (Cuadro 1). Los bosques secundarios como el bosque de pino-encino-liquidámbar (BPEL) y el bosque de pino (BP) tuvieron ligeramente una menor producción, pero significativamente más alta con respecto al bosque en proceso de regeneración (PR). Cabe indicar que esta última condición tiene la menor área basal como resultado de la

Cuadro 3. Relación comparativa de la producción de hojarasca $\left(\mathrm{Mg} \cdot \mathrm{ha}^{-1} \cdot \mathrm{año} \mathrm{O}^{-1}\right)$ entre los cinco tipos de vegetación evaluados en Chiapas y algunas comunidades forestales de México y de otras regiones del mundo.

\begin{tabular}{|c|c|c|c|}
\hline Tipo de Bosque & Localidad y (o) País & 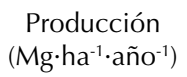 & Referencia \\
\hline Bosque de encino & Chiapas, México & 6.5 & Este estudio \\
\hline Bosque de pino-encino & & 7.5 & \\
\hline Bosque de pino & & 5.1 & \\
\hline Bosque de pino-encino-liquidámbar & & 5.3 & \\
\hline Parcelas en restauración & & 2.4 & \\
\hline Bosque mesófilo de montaña (BMM) & Xalapa, México & 8.45 & Williams-Linera y Tolome, 1996 \\
\hline Bosque lluvioso de montaña & Nueva Guinea & 7.6 & Edwards, 1977 \\
\hline BMM & Tamaulipas, México & 7.3 & Bracho y Puig, 1987 \\
\hline Bosque lluvioso de montaña & Colombia & $4.31-7.03$ & Veneklaas, 1991 \\
\hline BMM & Jamaica & 6.6 & Tanner, 1980 \\
\hline Bosque lluvioso de montaña & Venezuela & 4.3 & Tanner et al., 1992 \\
\hline Plantación de Pinus pinaster & España & 3.3 & Roig et al., 2005 \\
\hline Plantación de Pinus caribea & Nigeria & 5.7 & Egunjobi y Onweluzo, 1979 \\
\hline Bosque montano & Puerto Rico & 2.7 & Weaver et al., 1986 \\
\hline Bosques montanos & Hawai & $5.2-7.0$ & Vitousek et al., 1995 \\
\hline Bosque mixto de latifoliadas y coníferas & Nueva Zelanda & $6.7-8.7$ & Enright, 1999 \\
\hline Bosques caducifolios & India & $5.6-8.6$ & Sundarapandian y Swamy, 1999 \\
\hline Bosque perennifolio de neblina & Etiopía & 10.9 & Lisanework y Michelsen, 1994 \\
\hline
\end{tabular}


etapa de crecimiento en el que se encuentra caracterizada por la ausencia de árboles grandes. Este bosque está dominado por individuos de no más de 10 años de edad (DAP $<10 \mathrm{~cm}$ ) de especies de Pinus spp., Clethra suaveolens, Liquidambar styraciflua y varios individuos en su mayoría rebrotados de Quercus sapotifolia, que se han venido regenerando después de los incendios forestales ocurridos en el Parque de Montebello durante la estación seca de 1998. Aunque en estas últimas parcelas se han reintroducido poblaciones de 16 especies de árboles nativos, principalmente latifoliadas del interior y del dosel (Ortíz-Aguilar, 2006), su representación en la producción de hojarasca es aún incipiente.

Al comparar los valores de producción de hojarasca con otros estudios en comunidades forestales equivalentes (Cuadro 3), es posible observar que la producción total de hojarasca del BE y BPE está dentro de los valores más altos reportados en la literatura, aunque cabe mencionar que no siempre es posible comparar directamente con otros estudios debido a la alta variación interanual y a las propias diferencias en la estructura y composición del bosque. Por ejemplo, Edwards (1977), Sharma y Ambasht (1987) y Tanner (1980) separan los componentes en solo dos categorías: material leñoso y material no leñoso que a veces no son comparables ni entre ellas mismas ya que Tanner (1980) en su categoría de material leñoso incluye epífitas. Las categorías aplicadas en este trabajo se apegan más a las utilizadas por Williams-Linera y Tolome (1996) pero dividiendo la categoría de material reproductivo en flores y frutos para tener un mejor detalle de los datos. La mayoría de los estudios previos muestran que las hojas es el componente más representado dentro de la producción total de hojarasca (Cuadro 3).

En nuestro caso, el $72 \%$ del total de la hojarasca registrada en el BE correspondió solo a las hojas, valor muy semejante al 70\% reportado por Williams-Linera y Tolome (1996) en un bosque mesófilo de montaña de Veracruz. La producción máxima de material leñoso (ramas, corteza) en el presente trabajo fue de 5-12.5\% entre los tipos de vegetación, mientras que en otros estudios la contribución de esta fracción puede ser mayor. Sharma y Ambasht (1987) reportan 20\% de material leñoso en una plantación en el Himalaya; $18.9 \%$ en un bosque mixto en Hong Kong (Lam y Dudgeon, 1985), $17.6 \%$ en un bosque montano de Colombia (Veneklaas, 1991), $16 \%$ en un bosque tropical húmedo en Nueva Guinea (Edwards, 1977) y 15\% en un bosque mesófilo en Veracruz (Williams-Linera y Tolome, 1996). Sin embargo, estos porcentajes son muy variables debido a eventos esporádicos de caída de ramas o troncos o muerte prolongada de un árbol y que sus ramas o corteza caigan en las trampas de hojarasca.

En relación con las tasas de descomposición de la hojarasca de las especies analizadas, los resultados mostraron que en ningún caso ocurrió la desintegración completa de las muestras durante los primeros 300 días de evaluación. En este periodo, las muestras retuvieron entre un $35-60 \%$ de su peso inicial y no necesariamente los valores más bajos de descomposición se registraron en las parcelas de restauración, por lo que esta comunidad forestal parece tener las condiciones necesarias para que ocurra la descomposición de manera efectiva (figura 3). Aún considerando que nuestro diseño experimental no fue totalmente balanceado en cuanto al mismo número de especies dentro de cada tipo de bosque, los resultados si permiten distinguir una tendencia en la pérdida de peso a lo largo del tiempo, independientemente del tipo de vegetación y que Alnus acuminata subsp. arguta, una especie pionera, es la que presentó la mayor tasa de descomposición en los tres tipos de vegetación considerados (figuras 3 y 4).

Hay factores múltiples que afectan la velocidad de descomposición de la hojarasca, pero básicamente están controlados por tres factores interrelacionados: el clima, la calidad de la hojarasca y la abundancia de organismos degradadores (La Caro y Rudd, 1985; Coûteaux et al., 1995; Sundarapandian y Swamy, 1999; Loranger et al., 2002; Ostertag et al., 2003; Vasconcelos y Laurance, 2005; Sayer, 2006; Berg y McClaugherty, 2008). Brown y Lugo (1990) atribuyen una mayor descomposición a la actividad biológica presente en condiciones sucesionales tardías, dado que el bosque maduro tiene un reciclaje más eficiente de nutrientes y sus elementos en el suelo junto con la humedad y sus especies se encuentran más integrados. Negrete-Yankelevich (2004) al comparar la descomposición de una especie tardía (Persea americana Mill.) con una especie temprana (Pinus chiapensis (Martínez) Andresen) del bosque mesófilo en Oaxaca, no encontró diferencias en la descomposición entre diferentes condiciones sucesionales, pero $P$. americana se descompuso a una tasa mas baja que $P$. chiapensis. En nuestro estudio, no hubo una aparente diferencia en las tasas de descomposición entre la mayoría de las especies, a pesar de que se consideran sucesionalmente distintas (González-Espinosa et al., 2006). La excepción fue A. acuminata subsp. arguta, una especie típicamente pionera que tuvo consistentemente las mayores tasas de descomposición en los tres tipos de vegetación comparados. Los coeficientes de descomposición $(k)$ obtenidos en nuestro estudio son comparables a los reportados por Borders et al. (2006) y ligeramente más altos en comparación con otros ecosistemas forestales templados. Por ejemplo, Das y Ramakrishnan (1985) reportan para diferentes especies de pinos valores de $k$ entre 0.307 y 0.46 . Melillo et al. (1982) reportan para diferentes especies caducifolias de norteamérica valores entre 0.08 y 0.47 . Asimismo, Mo et al. (2007) registraron variaciones entre $0.24-0.30$ para poblaciones de Pinus massoniana D. Don en China. Sin embargo, nuestros resultados son más bajos cuando se comparan con otros ecosistemas tropicales húmedos (1.03-1.76 en Puerto Rico, Ostertag et al., 2003), o con diversos fragmentos forestales del bosque Amazónico (1.56-5.50, Didham, 1998) o con un bosque tropical subperennifolio en la Guayana Francesa (0.41-2.39, Loranger et al., 2002). 
Por otra parte, los componentes estructurales y nutricionales de la hojarasca son otros factores que determinan la tasa de descomposición (Zhang y Zak, 1995; Cleveland et al., 2006; Mo et al., 2007; Berg y McClaugherty, 2008) y al menos en estudios de corta duración se considera que la relación $\mathrm{C} / \mathrm{N}$ pronostica bien las tasas de descomposición de la hojarasca (Wieder y Lang, 1982; Tian et al., 1992; Xuluc-Tolosa et al., 2003; Alhamd et al., 2004). Por lo general, un bajo valor de $\mathrm{C} / \mathrm{N}$ anticipa una mayor tasa de descomposición. Los valores más bajos de la relación $\mathrm{C} / \mathrm{N}$ correspondieron para las especies que tuvieron una mayor tasa de descomposición (L. styraciflua y A. acuminata subsp. arguta), confirmado también con los valores más altos de $k$ (figura 4). En estudios de largo plazo, el papel del contenido de nutrientes es menos importante y la predicción de la pérdida de peso de la muestra puede realizarse directamente con el contenido de Carbono (Aber y Melillo, 1991). Los resultados de este estudio tienen importantes implicaciones para el manejo y restauración de bosques. Para propósitos de incrementar la materia orgánica de bosques secundarios, Babbar y Ewel (1989) han propuesto que las especies más lignificadas y menos lábiles son más apropiadas para disminuir parcialmente la tasa de liberación de nutrimentos del mantillo y ayudar a reducir el riesgo de pérdida por lixiviación. Con este criterio, proponemos que A. acuminata subsp. arguta y L. styraciflua pudiesen utilizarse en prácticas de enriquecimiento de bosques degradados de acuerdo con sus más altos niveles de incorporación de biomasa foliar. El uso de especies con hojas mas lignificadas como Chiranthodendron pentadactylon o Quercus crassifolia y $Q$. sapotifolia son recomendables para disminuir el riesgo de pérdida de elementos en el sistema al proteger el suelo del impacto directo de la lluvia y aumentar las posibilidades de almacenar elementos. Aunque por ahora únicamente se han presentado valores comparativos de la acumulación y desintegración de hojarasca entre distintos tipos de bosque, para que la restauración de forestal sea realmente efectiva hace falta diseñar nuevas investigaciones que incorporen un mayor número de especies y un mayor intervalo temporal que permita evaluar la descomposición de la hojarasca mediante la lixiviación de elementos, degradación por organismos edáficos y transformación de la materia orgánica por microorganismos y su mineralización, entre otros procesos (Lavelle et al. 1993; Berg y McClaugherty, 2008).

\section{Agradecimientos}

Agradecemos a las autoridades del Parque Nacional Lagunas de Montebello y a los habitantes de la comunidad de Merced Bazom por las facilidades para desarrollar el estudio. A Pedro Girón Hernandez, Elías Santiz López y Alfonso Luna Gómez por la ayuda durante las extenuantes jornadas de campo y a dos revisores anónimos por las observaciones y comentarios a una primera versión del manuscrito. Este estudio formó parte del proyecto "Definición de grupos funcionales vegetales para la restauración del bosque mesófilo de montaña en Chiapas" financiado por los fondos sectoriales de SEMARNAT-CONACYT (Clave SEMARNAT2002-01-C01-00048).

\section{Literatura citada}

Aber J.D. y Melillo J.M. 1991. Terrestrial Ecosystems, 2a . Edición. Academic Press, San Diego, California. 543 pp.

Aerts R. 1997. Climate, leaf litter chemistry and leaf litter decomposition in terrestrial ecosystems: a triangular relationship. Oikos 79:439-449.

Alhamd L., Arakaki S. y Hagihara A. 2004. Decomposition of leaf litter of four tree species in a subtropical evergreen broadleaved forest, Okinawa Island, Japan. Forest Ecology and Management 202:1-11.

Álvarez-Sánchez J. y Becerra E.R. 1996. Leaf decomposition in a Mexican tropical rain forest. Biotropica 28:657-667.

Anderson J.M. e Ingram J.S.I. 1993. Tropical soil biology and fertility: A hand book of methods. CAB International. Wallingford, Reino Unido. 171 pp.

Babbar L. y Ewel J. 1989. Descomposición del follaje en diversos ecosistemas sucesionales tropicales. Biotropica 21:20-29.

Bärlocher F. 2005. Leaf mass loss estimated by litter bag technique. En: Graca A.S., Bärlocher F. y Gessner M.O. Eds. Methods to study litter decomposition: a practical guide, pp. 36-42. Springer, Dordrecht, Alemania.

Berg B. y McClaugherty C. 2008. Plant litter: Decomposition, humus formation, Carbon sequestration, 2a edición. Springer, Berlin. 338pp.

Borders B.D., Pushnik J.C. y Wood. D.M. 2006. Comparison of leaf litter decomposition rates in restored and mature riparian forests on the Sacramento River, California. Restoration Ecology 14:308-315.

Bracho R. y Puig H. 1987. Producción de hojarasca y fenología de ocho especies importantes del estrato arbóreo. En: Puig H. y Bracho R. Eds. El Bosque Mesófilo de Montaña de Tamaulipas, pp. 81-106, Instituto de Ecología, A. C. Xalapa, Veracruz, México.

Brown S. y Lugo A.E. 1990. Tropical secondary forests. Journal of Tropical Ecology 6:1-32.

Camacho-Cruz A., González-Espinosa M., Wolf J.H.D. y de Jong B.H.J. 2000. Germination and survival of tree species in disturbed forests of the highlands of Chiapas, Mexico. Canadian Journal of Botany 78:1309-1318.

Cleveland C.C., Reed S.C. y Townsend A.R. 2006. Nutrient regulation of organic matter decomposition in a tropical rain forest. Ecology 87:492-503.

Coûteaux M-M., Bottner P. y Berg B. 1995. Litter decomposition, climate and liter quality. Trends in Ecology and Evolution 10:63-66.

Das A.K. y Ramakrishnan P.S. 1985. Litter dynamics in khasi pine (Pinus kesiya Royle ex Gordon) of north-eastern India. Forest Ecology and Management 10:135-153.

Del Valle-Arango J.I. 2003. Descomposición de la hojarasca fina en bosques pantanosos del Pacífico colombiano. Interciencia 28:148-153.

Didham R.K. 1998. Altered leaf-litter decomposition rates in tropical forest fragments. Oecologia 116:397-406. 
Edwards P.J. 1977. Studies of mineral cycling in a montane rain forest in New Guinea: II. The production and disappearance of litter. The Journal of Ecology 65:971-992.

Egunjobi J.K. y Onweluzo B.S. 1979. Litter fall, mineral turnover and litter accumulation in Pinus caribea L. stands at Ibadan, Nigeria. Biotropica 11:251-255.

Enright N.J. 1999. Litterfall dynamics in a mixed conifer-angiosperm forest in northern New Zealand. Journal of Biogeography 26:149-157.

Epstein H.E., Burke I.C. y Lauenroth W.K. 2002. Regional patterns of decomposition and primary production rates in the U.S. Great Plains. Ecology 83:320-327.

Galindo-Jaimes L., González-Espinosa M., Quintana-Ascencio P.F. y García-Barrios L. 2002. Tree composition and structure in disturbed stands with varying dominance by Pinus spp. in the highlands of Chiapas, Mexico. Plant Ecology 162:259-272.

Golicher D.J., Cayuela L., Alkemade J.R.M., González-Espinosa M. y Ramírez-Marcial N. 2008. Applying climatically associated species pools to the modeling of compositional change in tropical montane forests. Global Ecology and Biogeography 17:262-273.

González-Espinosa M., Quintana-Ascencio P.F., Ramírez-Marcial N. y Gaytán-Guzmán P. 1991. Secondary succession in disturbed Pinus-Quercus forests of the highlands of Chiapas, Mexico. Journal of Vegetation Science 2:351-360.

González-Espinosa M., Ramírez-Marcial N. y Galindo-Jaimes L. 2006. Secondary succession in Montane Pine-Oak Forests of Chiapas, México. En: Kappelle M. Ed. Ecology and Conservation of Neotropical Montane Oak Forests, pp. 209-221, Ecological Studies 185. Springer, Heidelberg.

La Caro F. y Rudd R.L. 1985. Leaf litter disappearance rates in Puerto Rican montane rain forest. Biotropica 17:269-276.

Lam P.K. y Dudgeon D. 1985. Seasonal effects on litterfall in a Hong Kong mixed forest. Journal of Tropical Ecology 1:55-64.

Lawrence D. 2005. Regional-scale variation in litter production and seasonality in tropical dry forests of southern Mexico. Biotropica 37:561-570.

Lavelle P., Blanchart E., Martin A., Martin S., Spain A., Toutain F., Barois I. y Schaefer R. 1993. A hierarchical model for decomposition in terrestrial ecosystems: application to soils of the humid tropics. Biotropica 25:130-150.

Lisanework N. y Michelsen A. 1994. Litterfall and nutrient release by decomposition in three plantations compared with a natural forest in the Ethiopian highland. Forest Ecology and Management 65:149-164.

Loranger G., Ponge J.F., Imbert D. y Lavelle P. 2002. Leaf decomposition in two semi-evergreen tropical forests: influence of litter quality. Biological Fertility of Soils 35:247-252.

Melillo J.M., Aber J.D., Muratore J.F. 1982. Nitrogen and lignin control of hardwood leaf litter decomposition dynamics. Ecology 63:621-626.

Mo J., Brown S., Xue J., Fang Y., Li Z., Li D. y Dong S. 2007. Response of nutrient dynamics of decomposing pine (Pinus massoniana) needles to simulated $\mathrm{N}$ deposition in a disturbed and a rehabilitated forest in tropical China. Ecological Research 22:649-658.

Negrete-Yankelevich S. 2004. Integrating soil macroinvertebrate diversity, litter decomposition and secondary succession in a tropical montane cloud forest in Mexico. Tesis doctoral, Universidad de Edimburgo, Escocia. 345 pp.
Olson J.S. 1963. Energy storage and the balance of producers and decomposers in ecological systems. Ecology 44:322-331.

Ortíz-Aguilar D.R. 2006. Plantaciones de enriquecimiento con árboles nativos para la restauración de un Bosque Mesófilo de Montaña en el Parque Nacional Lagunas de Montebello, Chiapas, México. Tesis de Licenciatura. Escuela de Biología. Universidad de Ciencias y Artes de Chiapas. Tuxtla Gutiérrez.

Ostertag R., Scatena F.N. y Silver W.L. 2003. Forest floor decomposition following hurricane litter inputs in several Puerto Rican forests. Ecosystems 6:261-273.

Quinn G. y Keough M. 2002. Experimental design and data analysis for biologists. 5ta ed. Cambridge University Press, Cambridge, Reino Unido.

R Development Core Team. 2008. R: a language and environment for statistical computing. R Foundation for Statistical Computing, Vienna (http://www.R-project.org).

Ramírez-Marcial N., Camacho-Cruz A., González-Espinosa M. 2005. Potencial florístico para la restauración de bosques en Los Altos y Montañas del Norte de Chiapas. En: González-Espinosa M., Ramírez-Marcial N. y Ruiz-Montoya L. Eds. Diversidad biológica en Chiapas, pp. 329-363. Plaza y Valdés, México, D.F.

Ramírez-Marcial N., Camacho-Cruz A., González-Espinosa M. y López-Barrera F. 2006. Establishment, survival and growth of tree seedlings under seccessional Montane Oak Forests in Chiapas, Mexico. En: Kappelle M. Ed. Ecology and Conservation of Neotropical Montane Oak Forests, pp. 177-189. Ecological Studies 185. Springer, Heidelberg.

Ramírez-Marcial N., González-Espinosa M. y Williams-Linera G. 2001. Anthropogenic disturbance and tree diversity in montane rain forests in Chiapas, Mexico. Forest Ecology and Management 154:311-326.

Rodríguez-Sánchez A.M.S. 2006. Composición florística y estructura de la vegetación en sitios bajo restauración ecológica del Parque Nacional Lagunas de Montebello. Tesis de Licenciatura, Centro Universitario de Ciencias Biológicas y Agropecuarias, Universidad de Guadalajara, Zapopan, Jalisco.

Roig S., del Río M., Cañellas I. y Montero G. 2005. Litter fall in Mediterranean Pinus pinaster Ait. stands under different thinning regimes. Forest Ecology and Management 206:179-190.

Sangha K.K., Jalota R.K. y Midmore D.J. 2006. Litter production, decomposition and nutrient release in cleared and uncleared pasture systems of central Queensland, Australia. Journal of Tropical Ecology 22:177-189.

Sayer E.J. 2006. Using experimental manipulation to assess the roles of leaf litter in the functioning of forest ecosystems. Biological Reviews 81:1-31.

Sharma E. y Ambasht R.S. 1987. Litterfall, decomposition and nutrient release in an age sequence of Alnus nepalensis plantation stands in the eastern Himalaya. The Journal of Ecology 75:997-1010.

Sundarapandian S.M. y Swamy P.S. 1999. Litter production and leaf-litter decomposition of selected tree species in tropical forests at Kodayar in the Western Ghats, India. Forest Ecology and Management 123:231-244.

Tanner E.V.J. 1980. Litterfall in montane rain forests of Jamaica and its relation to climate. The Journal of Ecology 68:833-848.

Tanner E.V.J., Kapos V. y Franco W. 1992. Nitrogen and Phosphorus fertilization effects on Venezuelan montane forest trunk growth and litterfall. Ecology 73:78-86. 
Tian G., Kang B.T., Brussaard L. 1992. Biological effects of plant residues with contrasting chemical compositions under humid tropical conditions-decomposition and nutrient release. Soil Biology and Biochemistry 24:1051-1060.

Vasconcelos H.L. y Laurance W.F. 2005. Influence of habitat, litter type, and soil invertebrates on leaf-litter decomposition in a fragmented Amazonian landscape. Oecologia 144:456-462.

Veneklaas E.J. 1991. Litterfall and nutrient fluxes in two montane tropical rain forests, Colombia. Journal of Tropical Ecology 7:319-336.

Vitousek P.M., Gerrish G., Turner D.R., Walker L.R y Mueller-Dombois D. 1995. Litterfall and nutrient cycling in four Hawaiian montane rainforests. Journal of Tropical Ecology 11:189-203.

Weaver P.L., Medina E., Pool D., Dugger K., Gonzales-Liboy J. y Cuevas E. 1986. Ecological observations in the dwarf cloud forest of the Luquillo Mountains in Puerto Rico. Biotropica 18:79-85.

Recibido: 8 de agosto de 2008

Aceptado: 21 de enero de 2009
Wedderburn M.E. y Carter J. 1999. Litter decomposition by four functional tree types for use in silvopastoral systems. Soil Biology and Biochemistry 31:455-461.

Wieder R.K. y Lang G.E. 1982. A critique of the analytical methods used in examining decomposition data obtained from litter bags. Ecology 63:1636-1642.

Williams-Linera G. y Tolome J. 1996. Litterfall, temperate and tropical dominant trees, and climate in a Mexican lower montane forest. Biotropica 28:649-656.

Xuluc-Tolosa F.J, Vester H.F.M., Ramírez-Marcial N., Castellanos-Albores J. y Lawrence D. 2003. Leaf litter decomposition of tree species in three successional phases of tropical dry secondary forest in Campeche, Mexico. Forest Ecology and Management 174:401-412.

Zhang Q. y Zak J.C. 1995. Effects of gap size on litter decomposition and microbial activity in a subtropical forest. Ecology 76:2196-2204. 